\title{
Electron solvation in methanol revisited
}

\author{
P. Holpár, T. Megyes, E. Keszei* \\ Department of Physical Chemistry, Eötvös University, P.O. Box. 32, H-1518, Budapest 112, Hungary
}

\begin{abstract}
Suggestions for the mechanism of electron solvation in methanol during the last three decades were mostly based on limited time resolution measurements, or indirect observations. The two-channel solvation scheme proposed by Lewis and Jonah (1986) based on indirect observations in electron scavenging experiments is checked here to see if it is in accordance with recent sub-picosecond pump-and-probe laser experimental results. We confirm the applicability of this solvation mechanism and calculate quantitative kinetic and spectral parameters involved. (C) 1999 Published by Elsevier Science Ltd. All rights reserved.
\end{abstract}

Keywords: Electron solvation; Ultrafast laser kinetics; Methanol

\section{Introduction}

Electron solvation in polar liquids has been an intriguing subject ever since the identification of the hydrated electron (Keene, 1960; Keene, 1963; Matheson, 1962; Hart and Boag, 1962). As its formation in liquid water was much too fast to experimentally follow, alcohols became ideal model solvents for the study of electron solvation kinetics. Many pulse radiolysis studies were made in alcohols cooled close to freezing point to sufficiently slow down the solvation process. Most of the suggested mechanistic ideas originate from studies done in the seventies (Baxendale and Wardman, 1971, 1973; Baxendale and Sharpe, 1976; Chase and Hunt, 1975; Klassen et al., 1975; Gilles et al., 1977; Okazaki and Freeman, 1978). A simple two-state stepwise mechanism interpreted the results in terms of two absorbing localized species: a "presolvated" and an "equilibrated" one. The blueshifting model contained one single localized species to

\footnotetext{
* Corresponding author. Tel.: + 36-1-209-0591; fax: + 36-1209-0602.

E-mail address: keszei@para.chem.elte.hu (E. Keszei)
}

absorb, whose spectrum temporarily shifted towards the blue wavelengths (Klassen et al., 1975; Gilles et al., 1977; Okazaki and Freeman, 1978). The combination of the two models was also proposed (Ogasawara et al., 1981). The advent of the sub-picosecond pumpand-probe laser technique allowed the study of electron hydration even in pure water (Migus et al., 1987; Long et al., 1990). However, the same technique applied to electron solvation in alcohols offered better insight into the slower solvation process (Hirata and Mataga, 1993; Walhout et al., 1995; Shi et al., 1995; Pépin et al., 1994). These laser studies enriched the mechanistic ideas and enabled quantitative testing of kinetic models. A detailed overview of different kinetic models can be found in a recent paper of Turi et al. (1997).

Turi et. al. (1997) have shown that a mechanism including thermalization and a subsequent branching localization (i.e., two localization channels) can describe the experimental data measured recently by Pépin et. al. (1994) for electron solvation in neat methanol, in addition to the interpretation on the basis of a blue-shifting hybrid model given by Pépin and coworkers themselves. Turi et. al. have stated that the branching thermalization mechanism carries many similarities to the mechanism proposed by Lewis and 
Jonah (1986). In this paper we report a quantitative analysis of observed data using the kinetic model of Lewis and Jonah.

The experimental data measured by Pépin et. al. (1994) comprise kinetic traces in an exceptionally wide wavelength range, from 400 to $1350 \mathrm{~nm}$, obtained following multiphoton ionization of pure methanol at $294 \mathrm{~K}$, with $625 \mathrm{~nm}(2 \mathrm{eV})$ laser pulses of approximately $300 \mathrm{fs}$ fwhm, with an irradiance of $\sim 10^{13} \mathrm{~W} / \mathrm{cm}^{2}$ inside the sample cell. Further details can be found in previous papers (Pépin et al., 1994; Turi et al., 1997).

Lewis and Jonah interpreted their electron scavenging experiments on the basis of the following mechanism:
Here, $e_{1}^{-}$to $e_{n}^{-}$are the thermalizing species with gradually less energy. The first electronic species that has lost enough energy so that it can be trapped is $e_{n}^{-}$, but it can still lose energy and get further thermalized to become $e_{\text {free}}^{-}$, which can also be trapped. However, there is a great difference between the two species; while the energetic $e_{n}^{-}$is directly trapped into a "final" solvated state, the less energetic $e_{\text {free }}^{-}$is trapped in a transient $e_{\text {tr }}^{-}$state, which relaxes to give the same final $e_{s}^{-}$. We would like to note that mechanism (2) is closely related to the one proposed by Turi et al. (1997), with the difference that the branching is from $e_{n}^{-}$here, and from $e_{\text {free }}$ there.

Supposing that all the elementary steps can be con-

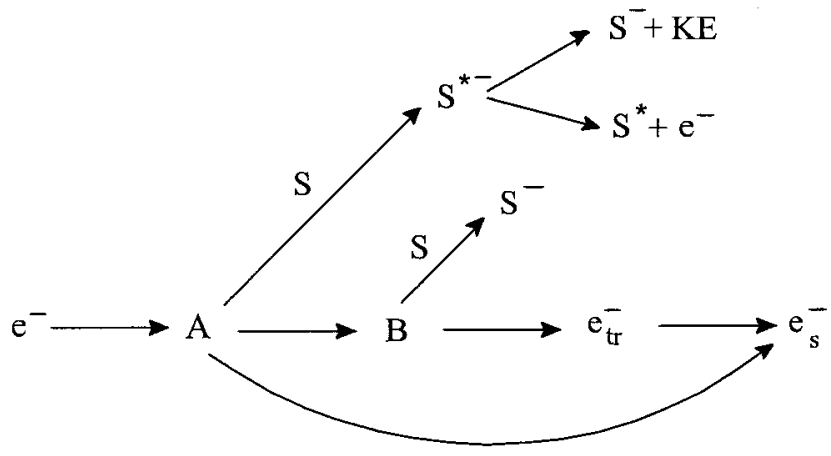

In this scheme the "horizontal" part from $e^{-}$to $e_{s}^{-}$ is the solvation mechanism, and state $\mathrm{A}$ has a higher (kinetic) energy than state $\mathrm{B}$, both being (nonlocalized) precursors of $e_{\mathrm{tr}}^{-}$and/or $e_{s}^{-}$. From the scavenging results ("vertical" part of the above mechanism) Lewis and Jonah concluded that there is a "fast solvation channel", A to $e_{s}^{-}$, and a "slow solvation channel", B to $e_{s}^{-}$via $e_{\mathrm{tr}}^{-}$, where $\mathrm{A}$ and $\mathrm{B}$ are both "dry" (i.e., nonlocalized) electrons. This idea has been put forward prior to the publication of the results of the first successful diabatic quantum dynamic electron hydration simulations (Webster et al., 1991; Murphrey and Rossky, 1993), and their kinetic analysis, which supported a mechanism much similar to scheme (1) (Keszei et al., 1993; Keszei et al., 1995).

To model the solvation in mechanism (1) we included a thermalization manifold previous to localization, an idea which emerged from the quantum dynamic simulations (Webster et al., 1991): sidered as unimolecular reactions and described by first-order kinetics - which is reasonable as the whole solvation is complete within 100 ps that would not allow bimolecular reactions to occur-we can solve the according system of differential equations. Having the solution, we can try to fit mechanism (2) to the experimental solvation kinetics.

\section{Results and discussion}

The system of differential equations corresponding to mechanism (2) is solved using a combination of the Laplace-transform method (Rodiguin and Rodiguina, 1964) and direct integration (Fraleigh, 1990). Details of the solution procedure and the actual concentration versus time functions can be found at the web-site http://femto.chem.elte.hu/MeOHsolvation/. To fit the

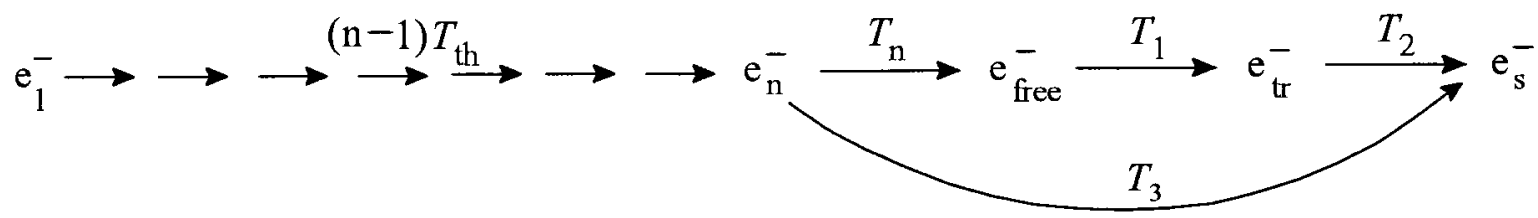


Table 1

Kinetic parameters of mechanism (2) for the solvation of electron in neat methanol. Numbers in parentheses indicate $95 \%$ confidence intervals. The overall thermalization time $\left(e_{1}^{-}\right.$to $\left.e_{\text {free }}^{-}\right)$is $n T_{\text {th }}$, where $n$ is the number of thermalization steps. The overall localization time $T_{\text {loc }}$ is the harmonic sum of $T_{1}$ and $T_{3}$, i.e. $1 /\left(1 / T_{1}+1 / T_{3}\right)$. The solvation time $\tau_{s}$ is the time when only $1 / e$ part of the electrons remains unsolvated (see Fig. 2). For comparison, the parameters of two recent models are also shown. $T_{\text {step }}$ is the equivalent of $T_{2}$ and $T_{\text {cont }}$ is the characteristic time of the spectral blue-shifts. All characteristic times are given in ps units. Note that the actual value of solvation time $\tau_{\mathrm{s}}$ largely depends on the kinetic model

\begin{tabular}{lccc}
\hline Parameter & Model (2) (present paper) & Turi et. al. (1997) & Pépin et. al. (1994) \\
\hline$n T_{\text {th }}$ & $0.50(0.02)$ & $0.56(0.01)$ & - \\
$T_{n}$ & $1.15(0.14)$ & - & - \\
$T_{1}$ & $5.73(0.19)$ & $5.9(0.31)$ & $T_{\text {step }}=6.1$ \\
$T_{2}$ & $10.23(0.74)$ & $8.4(0.40)$ & $T_{\text {cont }}=13.6$ \\
$T_{3}$ & $4.57(0.36)$ & $11.7(0.70)$ & $<1$ \\
$T_{\text {loc }}$ & 2.54 & 3.9 & 2.55 \\
$\tau_{S}$ & 15.6 & 10.3 & \\
\hline
\end{tabular}

obtained concentration function, the same reconvolution procedure was used as in Turi et al. (1997). We have found that mechanism (2) fits perfectly the experimental data at all 13 wavelengths analysed between 400 and $1350 \mathrm{~nm}$, which supports the applicability of the model.

The fitted kinetic parameters are summarized in Table 1. For comparison we also show kinetic parameters of the hybrid mechanism of Pépin et al. (1994) and that of Turi et al. (1997) with the shorter indirect solvation channel.

The reconstructed evolution of the electronic species using these parameters is shown in Fig. 1. From the figure we can see that the energetic species $e_{n}^{-}$disappears rather fast either by getting solvated to $e_{s}^{-}$, or by further loosing energy and becoming $e_{\overline{\text { free }}}$. This $e_{\overline{\text { free }}}^{-}$ species is still nonlocalized but it might be imagined as a quasi-free electron which is no more able to explore such a large surrounding than $e_{n}^{-}$does, so it cannot easily find a deep trap. Instead, it will get localized in

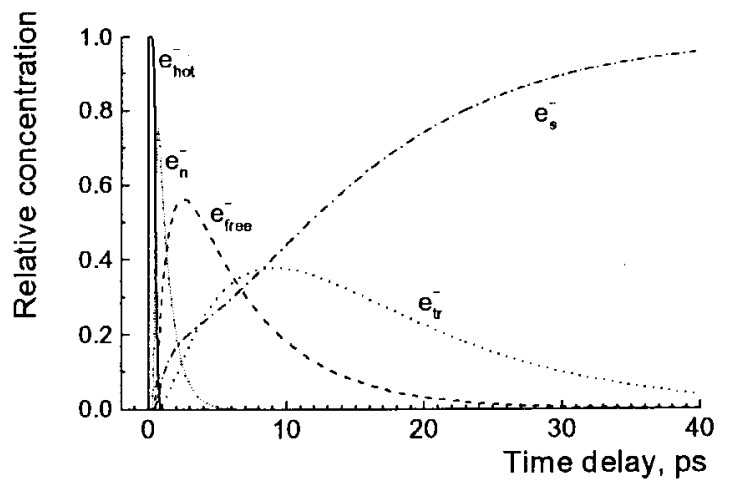

Fig. 1. Temporal evolution of the relative concentrations of the electronic species according to model (2). shallower traps to form $e_{\mathrm{tr}}^{-}$. This $e_{\mathrm{tr}}^{-}$then needs more time to relax into the final solvated state $e_{s}^{-}$.

From Fig. 2 the existence of a fast and slow solvation channel is clearly seen. The solvation time via the slow channel $(18.2 \mathrm{ps})$ is more than 12 times greater than that via the fast channel (1.45 ps), which confirms the original conjecture of Lewis and Jonah. We can also see from the figure that roughly $20 \%$ of the electrons become solvated directly (fast channel) and $80 \%$ via $e_{\text {free }}^{-}$and $e_{\text {tr }}^{-}$(slow channel). While the existence of these two channels explains the results found in the scavenging experiment (Lewis and Jonah, 1986), the great contribution of the slow channel and the relatively long persistence of the $e_{n}^{-} \rightarrow e_{\text {free }}^{-} \rightarrow e_{\text {tr }}^{-}$ series explains the observed large absorbances in the near-infrared-visible range during the first few picoseconds, and a gradual displacement of the transient spectra toward the blue region in the time-resolved sol-

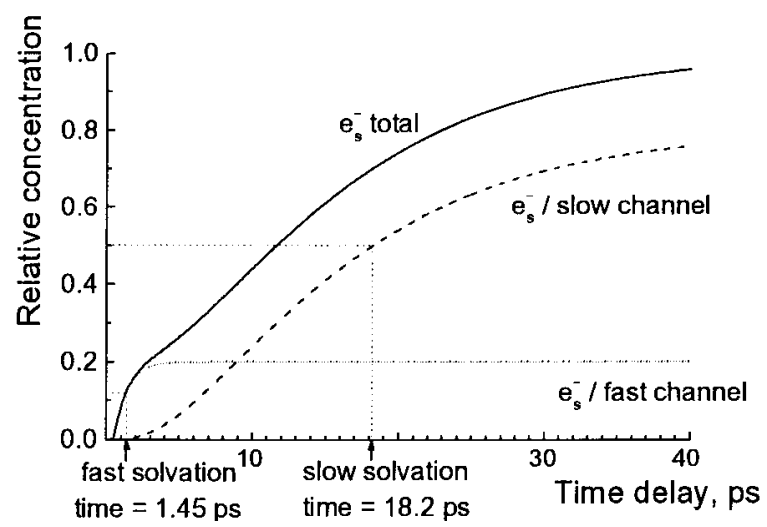

Fig. 2. Temporal evolution of the relative concentration of the solvated electrons formed in the slow and fast solvation channels. The curve corresponding to the overall solvation is labeled $e_{s}^{-}$total. The solvation time $\tau_{s}$ is the time when only $1 / e$ part of the electrons remains unsolvated. 


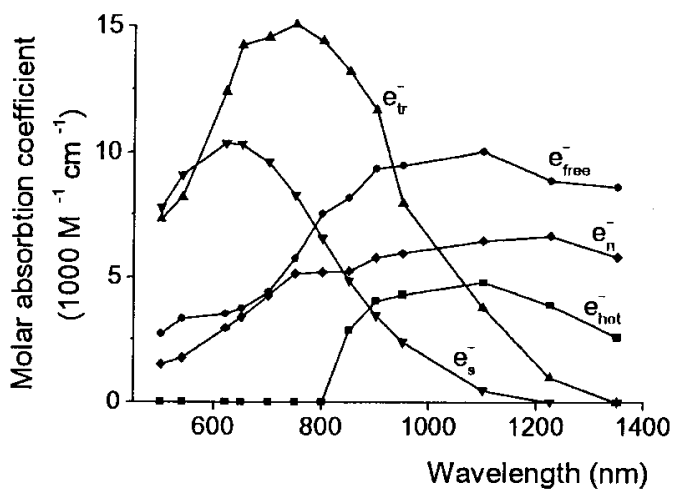

Fig. 3. Spectra of the five species included in mechanism (2) for electron solvation in neat methanol at room temperature. Spectral curves are labelled with the corresponding species. The spectrum of $e_{s}^{-}$is taken from the literature (Jou et al., 1977, 1979), while the other four are estimated from the present analysis. The spectrum labelled $e_{\text {hot }}^{-}$is an average assigned to all thermalizing species form $e_{1}^{-}$to $e_{n-1}^{-}$in mechanism (2).

vation experiment. The spectra of the five species included in model (2) are shown in Fig. 3. Comparing the spectra in the present paper to those published by Turi et al. (1997) for the mechanism with shorter indirect solvation, it can be observed that the spectra in Fig. 3 are smoother and the amplitude for $e_{\text {tr }}^{-}$is smaller. However, the spectral shape of the non-localized species $e_{n}^{-}$and $e_{\text {free }}^{-}$still extends to the visible wavelength range. (Similar spectra have been measured by Hase et al. for transient electronic species in glassy alcohols (Hase et al., 1972).) The smoother spectra found here suggest that electron solvation in methanol might proceed via model (2), rather than according to the shorter indirect solvation channel considered in the paper of Turi et al. (1997). As this latter model was found in dynamic simulations for water, structural and dynamic differences between water and methanol might explain the kinetic differences. Work is now in progress to perform the same type of quantum dynamic simulation for methanol as that has been done for water.

On the basis of the above observations we can conclude that the analysed experimental data support the suggestions of Lewis and Jonah concerning the fast and slow solvation channels. The main point of this work is that we have succeeded in calculating the kinetic and spectral parameters for a mechanism which has been conjectured based on indirect observations.

\section{Acknowledgements}

E. Keszei is a recipient of a grant support from the
National Research Fund of Hungary under Contract No. OTKA T019936. P. Holpár and T. Megyes acknowledge the support of the Ministry of Education and Culture in Hungary as Ph.D. students.

\section{References}

Baxendale, J.H., Wardman, P., 1971. Direct observation of solvation of the electron in liquid alcohols by pulse radiolysis. Nature 230, 449.

Baxendale, J.H., Wardman, P., 1973. Electrons in liquid alcohols at low temperatures. Journal of Chemical Society Faraday Transaction 1 69, 584.

Baxendale, J.H., Sharpe, P.H.G., 1976. A pulse radiolysis study of electrons in 1-propanol at low temperatures. International Journal of Radiation Physical Chemistry 8 , 621.

Chase, W.J., Hunt, J.W., 1975. Solvation time of the electron in polar liquids. Water and alcohols. Journal of Physical Chemistry 79, 2835.

Fraleigh, J.B., 1990. In: Calculus. Addison-Wesley, New York, p. 931.

Gilles, L., Bono, M.R., Schmidt, M., 1977. Electron in cold alcohols: a pulse radiolysis in ethanol. Canadian Journal Chemistry 55, 2003.

Hart, E.J., Boag, J.W., 1962. Journal of the American Chemical Society 84, 4090.

Hase, H., Warashina, T., Noda, M., Namiki, A., Higashimura, T., 1972. Trapped electrons produced in ethanol glass at $4 \mathrm{~K}$. Journal of Chemical Physics 57, 1039

Hirata, Y., Mataga, N., 1993. Electron photoejection and related phenomena in solutions - ultrafast laser photolysis studies. Progress in Reaction Kinetics 18, 273.

Jou, F.J., Freeman, G.R., 1977. Shapes of optical spectra of solvated electrons. Effect of pressure. Journal of Physical Chemistry 81, 909.

Jou, F.J., Freeman, G.R., 1979. Band resolution of optical spectra of solvated electrons in water, alcohols, and tetrahydrofuran. Canadian Journal of Chemistry 57, 591.

Keene, J.P., 1960. Nature 188, 843.

Keene, J.P., 1963. Nature 197, 47.

Keszei, E., Nagy, S., Murhprey, T.H., Rossky, P.J., 1993. Kinetic analysis of computer experiments on electron hydration dynamics. Journal of Chemical Physics 99, 2004.

Keszei, E., Murphrey, T.H., Rossky, P.J., 1995. Electron hydration dynamics: simulation results compared to pump and probe experiments. Journal of Physical Chemistry 99, 22 .

Klassen, N.V., Gillis, H.A., Teather, G.G., Kevan, L., 1975. Pulse radiolysis studies of time dependent spectral shifts of the solvated electron in ethanol and deuterated ethanol glasses at $76 \mathrm{~K}$. Journal of Chemical Physics 62, 2474.

Lewis, M.A., Jonah, C.D., 1986. Evidence for two electron states in solvation and scavening processes in alcohols Journal of Physical Chemistry 90, 5367.

Long, F.H., Lu, H., Eisenthal, K.B., 1990. Femtosecond studies of the presolvated electron: an excited state of the solvated electron. Physical Review Letters 64, 1469. 
Matheson, M.S., 1962. Annual Review of Physical Chemistry $13,77$.

Migus, A., Gauduel, Y., Martin, J.L., Antonetti, A., 1987. Excess electrons in liquid water: first evidence of a prehydrated state with femtosecond lifetime. Physical Review Letters 58, 1559.

Murphrey, T.H., Rossky, P.J., 1993. The role of solvent intramolecular modes in excess electron solvation dynamics. Journal of Chemical Physics 99, 515.

Ogasawara, M., Shimizu, K., Yoshida, H., 1981. Evolution of the spectra of the localized electrons in glassy alcohols irradiated at $4 \mathrm{~K}$. Radiation of Physical Chemistry 17, 331.

Okazaki, K., Freeman, G.R., 1978. Concerning the spectra and solvation process of electrons in liquid butanols. Canadian Journal of Chemistry 56, 2305.

Pépin, C., Goulet, T., Houde, D., Jay-Gerin, J.P., 1994. Femtosecond kinetic measurements of excess electrons in methanol: substantiation for a hybrid solvation mechanism. Journal of Physical Chemistry 98, 7009.

Rodiguin, N.M., Rodiguina, E.N., 1964. In: Schneider, R.F. (Ed.), Consecutive Chemical Reactions. D. van Nostrand Company, Princeton, New Jersey.

Shi, X., Long, F.H., Lu, H., Eisenthal, K.B., 1995. Electron solvation in neat alcohols. Journal of Physical Chemistry 99, 6917.

Turi, L., Holpár, P., Keszei, E., 1997. Alternative mechanisms for solvation dynamics of laser-induced electrons in methanol. Journal of Physical Chemistry 101, 5469.

Walhout, P.K., Alfano, J.C., Kimura, Y., Silva, C., Reid, P.J., Barbara, P.F., 1995. Direct pump/probe spectroscopy of the near-IR band of the solvated electron in alcohols Chemical Physics Letters 232, 135.

Webster, F.J., Schnitker, J., Friedrichs, M.S., Friesner, R.A., Rossky, P.J., 1991. Solvation dynamics of the hydrated electron: A nonadiabatic quantum simulation. Physical Review Letters 66, 3172. 